\title{
BMJ Open Differences in outcomes of patients with in-hospital versus out-of-hospital ST-elevation myocardial infarction: a registry analysis
}

\author{
Julia Stehli (D) , ${ }^{1,2}$ Misha Dagan, ${ }^{3}$ Diem T Dinh, ${ }^{4}$ Jeffrey Lefkovits, ${ }^{4,5}$ Ron Dick, ${ }^{2}$ \\ Stephanie Oxley, ${ }^{2}$ Angela L Brennan, ${ }^{4}$ Stephen J Duffy (iD , ${ }^{6}$ Sarah Zaman (i) ${ }^{7,8,9}$
}

To cite: Stehli J, Dagan M, Dinh DT, et al. Differences in outcomes of patients with in-hospital versus outof-hospital ST-elevation myocardial infarction: a registry analysis. BMJ Open 2022;12:e052000. doi:10.1136/ bmjopen-2021-052000

- Prepublication history and additional supplemental material for this paper are available online. To view these files, please visit the journal online (http://dx.doi.org/10.1136/ bmjopen-2021-052000).

Received 03 April 2021 Accepted 09 December 2021

Check for updates

(C) Author(s) (or their employer(s)) 2022. Re-use permitted under CC BY-NC. No commercial re-use. See rights and permissions. Published by BMJ.

For numbered affiliations see end of article.

Correspondence to Professor Sarah Zaman; sarah.zaman@sydney.edu.au

\section{ABSTRACT}

Objectives Patients with ST-elevation myocardial infarction (STEMI) that occur while already in hospital ('inhospital STEMI') face high mortality. However, data about this patient population are scarce. We sought to investigate differences in reperfusion and outcomes of in-hospital versus out-of-hospital STEMI.

Design, Setting and Participants Consecutive patients with STEMI all treated with percutaneous coronary intervention (PCl) across 30 centres were prospectively recruited into the Victorian Cardiac Outcomes Registry (2013-2018).

Primary and secondary outcomes Patients with in-hospital STEMI were compared with patients with out-of-hospital STEMI with a primary endpoint of 30-day major adverse cardiovascular events (MACE). Secondary endpoints included ischaemic times, all-cause mortality and major bleeding.

Results 0 f 7493 patients with PCl-treated STEMI, 494 $(6.6 \%)$ occurred in-hospital. Patients with in-hospital STEMI were older ( 67.1 vs 62.4 years, $p<0.001$ ), more often women ( $32 \%$ vs $19.9 \%, p<0.001)$, with more comorbidities. Patients with in-hospital STEMI had higher 30-day MACE $(20.4 \%$ vs $9.8 \%, p<0.001)$, mortality $(12.1 \%$ vs $6.9 \%, p<0.001)$ and major bleeding $(4.9 \%$ vs $2.3 \%, p<0.001)$, than patients with out-of-hospital STEMI. According to guideline criteria, patients with in-hospital STEMI achieved symptom-to-device times of $\leq 70 \mathrm{~min}$ and $\leq 90 \mathrm{~min}$ in $29 \%$ and $47 \%$, respectively. Patients with out-of-hospital STEMl achieved door-to-device times of $\leq 90 \mathrm{~min}$ in $71 \%$. Occurrence of STEMI while in hospital independently predicted higher MACE (adjusted OR 1.77, $95 \% \mathrm{Cl} 1.33$ to $2.36, p<0.001$ ) and 12 -month mortality (adjusted OR 1.49, 95\% Cl 1.08 to 2.07, $\mathrm{p}<0.001$ ).

Conclusions Patients with in-hospital STEMI experience delays to reperfusion with significantly higher MACE and mortality, compared with patients with out-of-hospital STEMI, after adjustment for confounders. Focused strategies are needed to improve recognition and outcomes in this high-risk and understudied population.

\section{INTRODUCTION}

Coronary artery disease is the leading cause of death worldwide. ${ }^{1}$ In particular, patients with ST-elevation myocardial infarction
Strengths and limitations of this study

- Patients with in-hospital ST-elevation myocardial infarction (STEMI) face high mortality. However, data about this patient population are scarce. We were the first to compare consecutive patients with in-hospital to out-of-hospital STEMI, who were all treated with percutaneous coronary intervention $(\mathrm{PCl})$ and were prospectively recruited into the Victorian Cardiac Outcomes Registry.

- Patients with in-hospital STEMI achieved symptomto-device times of $\leq 90 \mathrm{~min}$ and $\leq 70 \mathrm{~min}$ in only $47 \%$ and $29 \%$, respectively. They were less likely to receive guideline-directed medical therapy than standard out-of-hospital STEMls. Occurrence of STEMI while in-hospital independently predicted higher adjusted major adverse cardiovascular events and 12-month mortality.

- Our study provides observational data and therefore only associations can be made between ischaemic time and outcomes.

- Our study is limited by the registry not capturing the condition for which patients with in-hospital STEMI were hospitalised, which could have influenced outcomes.

- Our registry only captures patients with STEMI treated with $\mathrm{PCl}$; hence, we cannot comment on differences of all patients with STEMI who did not undergo revascularisation.

(STEMI) experience high mortality, despite advancements in treatment. ${ }^{2}$ Timely revascularisation with percutaneous coronary intervention (PCI) is a critical component of improving STEMI outcomes, ${ }^{3}$ with ischaemic time directly linked to mortality. ${ }^{45}$ STEMIs that occur in patients already hospitalised for an alternate condition, termed 'in-hospital STEMIs', have different characteristics and outcomes to classical out-of-hospital STEMIs. Patients with in-hospital STEMI are older, more often women with higher coagulopathy and lower occurrence of typical symptoms. ${ }^{6}$ 
While outcomes of out-of-hospital STEMIs have significantly improved over the last decade, ${ }^{7}$ mortality has remained constantly high for in-hospital STEMIs. ${ }^{89}$ This issue has never been more important than during the era of a COVID-19 pandemic. While out-of-hospital STEMI numbers have declined in COVID-19 affected areas, ${ }^{10}$ in-hospital STEMIs have been increasingly reported, with COVID-19 associated with STEMI mimickers, acute thrombosis and plaque rupture. ${ }^{1112}$

Despite the high mortality observed for in-hospital STEMIs, less than a handful of studies have specifically assessed this STEMI population. The studies that have been performed are limited by their single-centre nature, and/or inclusion of a heterogenous in-hospital STEMI population of which a large proportion did not undergo invasive angiography. ${ }^{78}$ Hence, the reasons for higher mortality of patients with in-hospital STEMI have not been fully elucidated. The contribution of system-wide delays in reperfusion and gaps in guideline-directed medical management may play a role. We need more research into STEMIs that occur while in-hospital, if we are to improve outcomes in this understudied population. This first-of-itskind Australian study used a large, multicentre, prospective PCI registry with the aim of investigating differences in ischaemic times, management and outcomes of in-hospital versus patients with out-of-hospital STEMI.

\section{METHODS}

\section{Study design and patient population}

From 2013 to 2018, consecutive patients with STEMI treated with PCI were prospectively recruited into the Victorian Cardiac Outcomes Registry. The Victorian Cardiac Outcomes Registry is an Australian, state-based clinical quality registry designed to monitor the performance and outcome of PCI in Victoria. It was established in 2012 and is engaged at all Victorian hospitals (13 public (ie, government funded) and 17 private) with all patients undergoing PCI or attempted PCI entered into the registry. ${ }^{13}$ It collects baseline demographic, procedural characteristics, in-hospital and 30-day outcomes through a secure web-based data collection system. ${ }^{14}$ Data integrity is ensured with regular audit activities conducted by the central registry. The Victorian Cardiac Outcomes Registry is funded by the Victorian Department of Health and Human Services. It obtains 12-month mortality for all patients through linkage with the Australian National Death Index. The deidentified data analysed for the purpose of this study are available on request to the Victorian Cardiac Outcomes Registry Committee (email: vcor@monash.edu).

\section{Inclusion and exclusion criteria}

Consecutive patients with STEMI who received successful or attempted PCI were included. At the time of entering the patient into the Victorian Cardiac Outcomes Registry database, STEMI was defined as elevated cardiac biomarkers and new ST-segment elevation in two or more contiguous leads. The clinical definition was based on a maximal concentration of troponin $\mathrm{T}$ or I above the MI diagnostic limit on at least one occasion within 24 hours from the index clinical event and ST-segment elevation in the ECG, equal to the Fourth Universal Definition of Myocardial Infarction. ${ }^{15}$ In-hospital STEMI was defined and captured as per Victorian Cardiac Outcomes Registry definition, based on the timing of symptom onset occurring during a hospital admission. The time recorded for symptom onset was when the patients advised the hospital staff of their symptoms. Out-of-hospital STEMI included all other STEMI cases where symptom onset occurred in the community setting. The in-hospital STEMI cohort was compared with the out-of-hospital STEMI cohort. For the analysis of symptom-to-device (STD) and door-todevice (DTD) time, the following patients were excluded from the analysis: (1) patients without a recorded time of symptom onset, (2) patients $>12$ hours from symptom onset, (3) patients who had symptom onset while admitted to a non-PCI capable hospital (for the in-hospital STEMIs), and (4) patients who presented to a nonPCI capable hospital (for the out-of-hospital STEMIs, outlined in figure 1).

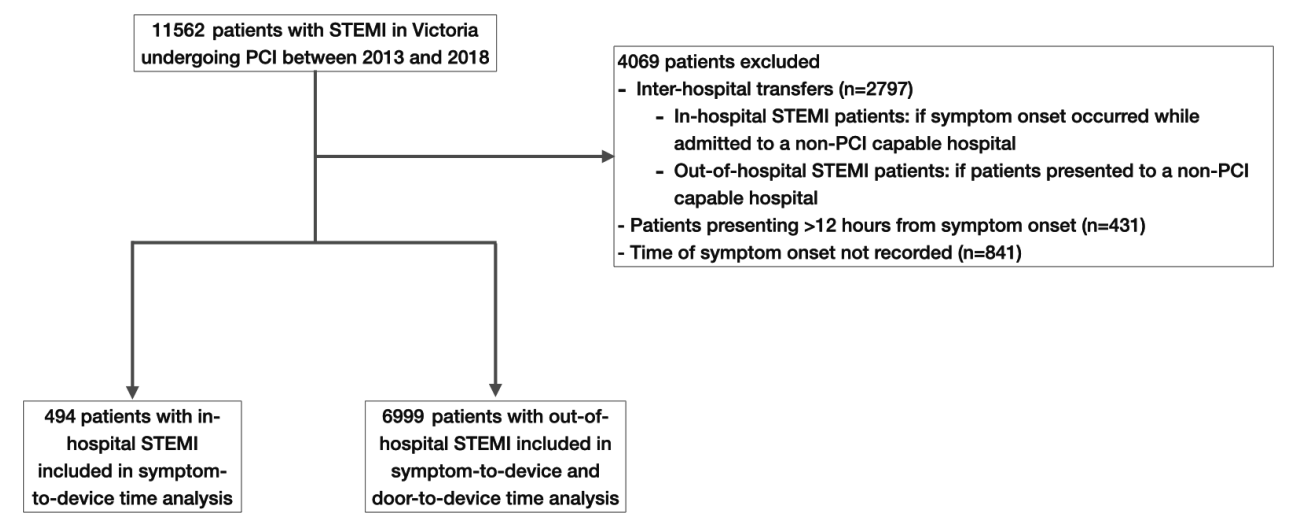

Figure 1 Inclusion of patients with in-hospital and out-of-hospital ST-elevation myocardial infarction (STEMI) into the timeanalysis. Flow chart showing patients which patients were excluded from and included into the analysis of symptom-to-device and door-to-device time. $\mathrm{PCl}$, percutaneous coronary intervention. 


\section{Primary and secondary outcomes}

The primary endpoint was 30-day major adverse cardiovascular events (MACE, consisting of all-cause death, new or recurrent MI, stent thrombosis or target vessel revascularisation). Secondary endpoints included STD and DTD times, 30-day and 12-month all-cause mortality, major adverse cardiovascular and cerebrovascular events (MACCE, consisting of MACE and stroke), major bleeding (consisting of type 3 and 5 according to the Bleeding Academic Research Consortium definition), recurrent MI, new heart failure (defined according to clinical signs), new renal impairment, length of hospital stay and referral to cardiac rehabilitation. STD time was calculated for patients with in-hospital STEMI. DTD time was calculated for patients with out-of-hospital STEMI.

The European Society of Cardiology guidelines recommend a time of $\leq 60 \mathrm{~min}$ from STEMI diagnosis to wire crossing in patients presenting to a PCI-capable hospital. ${ }^{4}$ Additionally, first medical contact (FMC)-to-ECG acquisition time is recommended at $\leq 10 \mathrm{~min} .{ }^{4}$ As there are no guidelines specific to in-hospital STEMIs, the time of symptom onset (as patients were already admitted under medical care) was used to take the place of FMC. An FMCto-ECG acquisition time of $\leq 10 \mathrm{~min}$ and a STEMI diagnosis to wire crossing time of $\leq 60 \mathrm{~min}$ would result in an ideal STD time of $\leq 70 \mathrm{~min}$ for in-hospital STEMIs. Accordingly, to assess for reperfusion delays, the percentage of patients with in-hospital STEMI achieving an STD time $\leq 70 \mathrm{~min}$ was analysed. The American Heart Association/American College of Cardiology guidelines recommend an FMC-to-device time of $\leq 90 \mathrm{~min}$ in patients presenting to a PCI-capable hospital. ${ }^{5}$ Accordingly, to assess for reperfusion delays the percentage of patients with in-hospital STEMI achieving a STD time $\leq 90 \mathrm{~min}$ and the percentage and patients with out-of-hospital STEMI achieving a DTD time $\leq 90 \mathrm{~min}$ was assessed. The terms 'device time' and 'wire crossing time' were used interchangeably for reperfusion time.

Preprocedural creatinine was collected up to 60 days before the PCI and the Cockcroft-Gault formula used to determine estimated glomerular filtration rate. New renal impairment was defined as an absolute rise of serum creatinine $\geq 44.2 \mu \mathrm{mol} / \mathrm{L}$ or $\geq 25 \%$ up to 5 days after the index PCI, when compared with baseline creatinine. Left ventricular ejection fraction was collected during the index STEMI admission or up to 30 days post discharge. Moderate-severe left ventricular dysfunction was defined as left ventricular ejection fraction $<45 \%$. New heart failure was defined as clinical evidence of heart failure. Complex lesions included type B2 and C lesions according to the American Heart Association/American College of Cardiology classification. Mechanical ventricular support included intra-aortic balloon pump, left ventricular assist device and/or extracorporeal membrane oxygenation.

\section{Statistical analysis}

Categorical variable associations were analysed using a $\chi^{2}$ or Fisher's exact test, as appropriate, and expressed as number/percentage. Continuous variables were analysed with a t-test and expressed as mean/SD. Univariable and multivariable associations with type of STEMI were determined by logistic regression. As the ischaemic time spans were highly skewed, their data were log-transformed for analysis and back to determine an estimated geometric mean. Along with type of STEMI, variables determined a priori to be included in the multivariable models were age, diabetes, estimated glomerular filtration rate, previous PCI and/or coronary artery bypass grafting, history of peripheral vascular disease and cerebrovascular disease, left ventricular ejection fraction, cardiogenic shock or cardiac arrest (out-of-hospital cardiac arrest for patients with out-of-hospital STEMI) requiring intubation, occurrence time of symptom onset, stent thrombosis or vein graft lesion. The variables forced into and retained in the model were determined based on prior literature ${ }^{16}$ and experience that these factors are known to influence MACE and all-cause mortality. Statistical analyses were performed using Stata V.14 with $p$ value $<0.05$ considered statistically significant.

\section{Patient and public involvement}

Neither patients nor the public were involved in this retrospective analysis.

\section{RESULTS}

\section{Baseline characteristics}

A total of 7493 patients underwent PCI for the treatment of STEMI of which 494 (6.6\%) were in-hospital STEMI. Baseline demographic and clinical characteristics are shown in table 1. Patients with in-hospital versus outof-hospital STEMI were significantly older, more often women, with more comorbidities and higher proportions with cardiogenic shock or cardiac arrest requiring intubation.

\section{Procedural and discharge characteristics}

Stent thrombosis was more frequently seen for in-hospital versus out-of-hospital STEMIs. Of stent thromboses resulting in an in-hospital STEMI, 5\% (4/82) occurred during the same admission as an initial PCI. Patients with in-hospital STEMI needed more mechanical support, with radial access used less frequently than for out-ofhospital STEMIs. Patients with in-hospital STEMI were significantly less likely to receive guideline-directed medical therapy (adjusted OR $0.70,95 \%$ CI 0.56 to 0.87 , $\mathrm{p}<0.001$, table 2).

\section{Ischaemic times}

Patients with in-hospital STEMI achieved an STD time of $\leq 70 \mathrm{~min}^{4}$ in $29 \%$ of cases and a STD time $\leq 90 \mathrm{~min}^{5}$ in $47 \%$ of cases (figure 2). Correspondingly, patients with out-of-hospital STEMI achieved a DTD time of $\leq 90 \mathrm{~min}^{5}$ in $71 \%$ of cases.

In the in-hospital STEMI group, unadjusted and adjusted geometric mean STD times were $110 \mathrm{~min}$ and 
Table 1 Baseline and clinical characteristics according to patients with in-hospital versus out-of-hospital ST-elevation myocardial infarction (STEMI)

\begin{tabular}{|c|c|c|c|c|}
\hline & \multirow{2}{*}{$\begin{array}{l}\text { Total patients with STEMI } \\
\mathrm{n}=7493\end{array}$} & \multirow{2}{*}{$\begin{array}{l}\text { In-hospital STEMI } \\
\mathrm{n}=494\end{array}$} & \multirow{2}{*}{$\begin{array}{l}\text { Out-of-hospital STEMI } \\
\mathrm{n}=6999\end{array}$} & \multirow[b]{2}{*}{$P$ value } \\
\hline & & & & \\
\hline Age (years) & $62.7 \pm 12.7$ & $67.1 \pm 12.7$ & $62.4 \pm 12.6$ & $<0.001$ \\
\hline Females, n (\%) & $1552(20.7 \%)$ & $158(32.0 \%)$ & 1394 (19.9\%) & $<0.001$ \\
\hline BMI $\left(\mathrm{kg} / \mathrm{m}^{2}\right)$ & $27.5[24.6-30.7)$ & $27.7[24.7-31.2)$ & $27.5[24.6-30.6)$ & 0.30 \\
\hline Diabetes, n (\%) & 1201 (16.0\%) & 111 (22.5\%) & 1090 (15.6\%) & $<0.001$ \\
\hline $\begin{array}{l}\text { eGFR }(\mathrm{ml} / \mathrm{min})<45 \mathrm{~mL} / \mathrm{min} \text {, } \\
\mathrm{n}(\%)\end{array}$ & $578(9.4 \%)$ & $78(16.4 \%)$ & $500(8.8 \%)$ & $<0.001$ \\
\hline $\begin{array}{l}\text { Moderate-severe LVEF } \\
\text { impairment, } \mathrm{n}(\%)\end{array}$ & 2029 (27.0\%) & $128(29.0 \%)$ & 1901 (28.9\%) & 0.965 \\
\hline $\begin{array}{l}\text { Previous CABG, and/or PCI, } \\
n(\%)\end{array}$ & $1196(15.9 \%)$ & 231 (46.7\%) & 965 (13.7\%) & $<0.001$ \\
\hline $\begin{array}{l}\text { Cerebrovascular disease, } \mathrm{n} \\
\text { (\%) }\end{array}$ & 256 (3.4\%) & $35(7.1 \%)$ & $221(3.2 \%)$ & $<0.001$ \\
\hline $\begin{array}{l}\text { Peripheral vascular disease, } \\
\text { n (\%) }\end{array}$ & $165(2.2 \%)$ & 36 (7.3\%) & $129(1.8 \%)$ & $<0.001$ \\
\hline $\begin{array}{l}\text { Oral anticoagulant therapy, } \\
\mathrm{n}(\%)\end{array}$ & $211(2.8 \%)$ & 31 (6.3\%) & $180(2.6 \%)$ & $<0.001$ \\
\hline $\begin{array}{l}\text { Onset of symptoms 07:00- } \\
20: 00, n(\%)\end{array}$ & $4832(64.7 \%)$ & 298 (62.7\%) & $4534(64.8 \%)$ & 0.37 \\
\hline $\begin{array}{l}\text { Cardiogenic shock or cardiac } \\
\text { arrest requiring intubation, } \\
\mathrm{n}(\%)\end{array}$ & $872(11.6 \%)$ & 77 (15.6\%) & 795 (11.4\%) & 0.005 \\
\hline $\begin{array}{l}\text { Prehospital ECG notification, } \\
\mathrm{n}(\%)\end{array}$ & $3993(57.0 \%)$ & - & 3993 (57.0\%) & \\
\hline
\end{tabular}

Values are number (\%) or median (IQR) or mean \pm SDs.

$\mathrm{BMI}$, body mass index; CABG, coronary artery bypass grafting; eGFR, estimated glomerular filtration rate; LVEF, left ventricular ejection fraction; $\mathrm{PCl}$, percutaneous coronary intervention.;

80 min, respectively. Within the in-hospital STEMI group, patients with stent thrombosis $(\mathrm{n}=82)$ had a significantly shorter geometric mean STD time than patients without stent thrombosis (unadjusted: 83 vs $116 \mathrm{~min}, \mathrm{p}=0.003$; adjusted: 68 vs $96 \mathrm{~min}, \mathrm{p}=0.008$, not shown in table).

\section{Clinical outcomes}

30-day and 12-months clinical outcomes are shown in table 3 .

Thirty-day MACE was significantly higher for in-hospital versus out-of-hospital STEMIs (20.4\% vs 9.8\%, $\mathrm{p}<0.001)$ with an adjusted OR of 1.77 (95\% CI 1.33 to $2.36, \mathrm{p}<0.001)$. Twelve-month mortality was significantly higher for patients with in-hospital versus out-ofhospital STEMI $(22.5 \%$ vs $11.2 \%, \mathrm{p}<0.001)$, adjusted OR 1.49 (95\% CI 1.08 to 2.07, $<<0.001$ ). Figure 3 shows unadjusted and adjusted Kaplan-Meier survival analysis. In-hospital symptom onset was an independent predictor for both 30-day MACE (adjusted OR 1.91, 95\% CI 1.38 to 2.64, $\mathrm{p}<0.001$ ) and 12-month mortality (adjusted OR $1.49,95 \%$ CI 1.08 to $2.07, \mathrm{p}<0.001)$. Independent multivariable associations with 30-day MACE and 12-month mortality are shown in the online supplemental table 1.

\section{DISCUSSION}

In this large, Australian PCI registry study, we investigated differences in presentation, treatment and outcomes of STEMI occurring in a patient already admitted to hospital, as compared with out-of-hospital STEMI. The principal findings of our study were that patients with in-hospital STEMI were (1) older, more likely to be women and have more comorbidities, (2) more likely to experience delays in reperfusion, (3) less likely to receive guideline-directed medical therapy, and (4) significantly more likely to experience MACE with higher all-cause mortality, compared with patients with out-of-hospital STEMI. After adjustment for confounders, patients who experienced a STEMI while already admitted to hospital had $91 \%$ higher odds of 30-day MACE and $88 \%$ higher odds of 12-month mortality, compared with standard STEMIs.

Patients with in-hospital STEMI were found to comprise $6.6 \%$ of overall STEMIs, similar to previously described. ${ }^{81718}$ While this percentage may not appear large, the chance of a STEMI occurring while inpatient is 40-50-fold that seen in the general community. ${ }^{19}{ }^{20}$ This is the first Australian study to assess in-hospital STEMIs 
Table 2 Procedural and discharge characteristics according to patients with in-hospital versus out-of-hospital ST-elevation myocardial infarction (STEMI)

\begin{tabular}{|c|c|c|c|}
\hline & In-hospital STEMI & Out-of-hospital STEMI & \multirow[b]{2}{*}{$P$ value } \\
\hline & $\mathrm{n}=494$ & $\mathrm{n}=6999$ & \\
\hline Radial access, $n$ (\%) & $185(37.4 \%)$ & 3850 (55.0\%) & $<0.001$ \\
\hline Glycoprotein Ilb/IIla inhibitor, n (\%) & $166(33.6 \%)$ & 2655 (37.9\%) & 0.055 \\
\hline Mechanical ventricular support, n (\%) & $44(8.9 \%)$ & $220(3.1 \%)$ & $<0.001$ \\
\hline \multicolumn{4}{|l|}{ Culprit vessel, n (\%) } \\
\hline RCA & 202 (40.9\%) & 2851 (40.7\%) & \multirow[t]{5}{*}{$<0.001$} \\
\hline LAD & $206(41.7 \%)$ & $2914(41.6 \%)$ & \\
\hline LCx & $61(12.3 \%)$ & $1108(15.8 \%)$ & \\
\hline Left main & $9(1.8 \%)$ & $89(1.3 \%)$ & \\
\hline Graft & $16(3.2 \%)$ & $37(0.5 \%)$ & \\
\hline Stent thrombosis, n (\%) & $82(16.6 \%)$ & $217(3.1 \%)$ & $<0.001$ \\
\hline Complex lesion, n (\%) & $366(74.1 \%)$ & $4628(66.1 \%)$ & $<0.001$ \\
\hline Number of stents implanted & $1.09 \pm 0.65$ & $1.17 \pm 0.52$ & 0.002 \\
\hline Drug-eluting stent, $\mathrm{n}(\%)$ & $344(69.6 \%)$ & $5336(76.2 \%)$ & $<0.001$ \\
\hline Procedural success, n (\%) & 461 (93.3\%) & $6610(94.4 \%)$ & 0.30 \\
\hline Length of stay & $9.7 \pm 19.1$ & $4.9 \pm 4.7$ & $<0.001$ \\
\hline Referral to cardiac rehabilitation, $\mathrm{n}(\%)$ & $327(74.7 \%)$ & $5538(84.4 \%)$ & $<0.001$ \\
\hline \multicolumn{4}{|l|}{ Discharge medications, $\mathrm{n}(\%)$} \\
\hline Aspirin & $415(95.2 \%)$ & 6409 (98.1\%) & $<0.001$ \\
\hline Thienopyridine & $153(35.1 \%)$ & $1802(27.6 \%)$ & $<0.001$ \\
\hline Ticagrelor & $267(61.4 \%)$ & $4606(70.5 \%)$ & $<0.001$ \\
\hline Beta blockers & $338(78.1 \%)$ & $5735(87.9 \%)$ & $<0.001$ \\
\hline ACE/ARB & $307(70.7 \%)$ & $5526(84.7 \%)$ & $<0.001$ \\
\hline Statin & 401 (92.4\%) & $6338(97.1 \%)$ & $<0.001$ \\
\hline Oral anticoagulants & 49 (11.3\%) & $561(8.6 \%)$ & 0.054 \\
\hline
\end{tabular}

Values are number (\%) or mean \pm SD.

ARB, angiotensin receptor blockers; LAD, left anterior descending artery; LCx, circumflex artery; RCA, right coronary artery.;

and similar to the few previous studies ${ }^{817} 18$ patients with in-hospital STEMI had significantly higher mortality, MACE, MACCE, major bleeding, recurrent MI and new renal impairment; all were approximately double that of out-of-hospital STEMIs, with recurrent MI threefold higher. These striking differences persisted after adjustment for confounders. It is important to note that previous studies investigating in-hospital STEMIs,${ }^{18}$ including the largest one by Kaul et $a l^{8}{ }^{8}$ assessed a heterogenous population with a large proportion who did not undergo PCI. From these studies, we know that patients with in-hospital STEMI receive less invasive angiography, potentially negatively influencing the outcomes. ${ }^{78}$ This is the first study to demonstrate significantly poorer outcomes for in-hospital STEMIs who were all deemed suitable for primary PCI.

Of course, in-hospital STEMIs are a distinct group of patients: being older, more often women, with higher comorbidities and complex coronary lesions. In addition, the underlying reason for hospitalisation may bias against the early recognition of STEMI and possibly affect clinical care decisions. Previous registry studies have shown that $34 \%-41 \%$ of in-hospital STEMIs occur in patients hospitalised for non-cardiac reasons, with the remainder occurring in cardiac inpatients. ${ }^{17} 21$ However, only a single study has adjusted for admission reason, and still found a significantly higher mortality for in-hospital compared with patients with out-of-hospital STEMI. ${ }^{8}$

Poorer outcomes of in-hospital STEMIs may in part be due to reperfusion delays. According to the European Society of Cardiology guidelines, a symptom-onsetto-ECG acquisition and STEMI-diagnosis-to-reperfusion of $\leq 10$ and $\leq 60 \mathrm{~min},{ }^{4}$ respectively, are recommended. Yet despite symptom onset within a PCI-capable hospital, less than a third of our in-hospital STEMIs achieved this. The American Heart Association/American College of Cardiology recommend an STD time of $\leq 90 \mathrm{~min}^{5}$; yet this was achieved in less than half of our patients with in-hospital STEMI. We know from previous in-hospital STEMI studies that the majority of ischaemic delays occur from symptom onset to catheterisation laboratory activation, presumably 


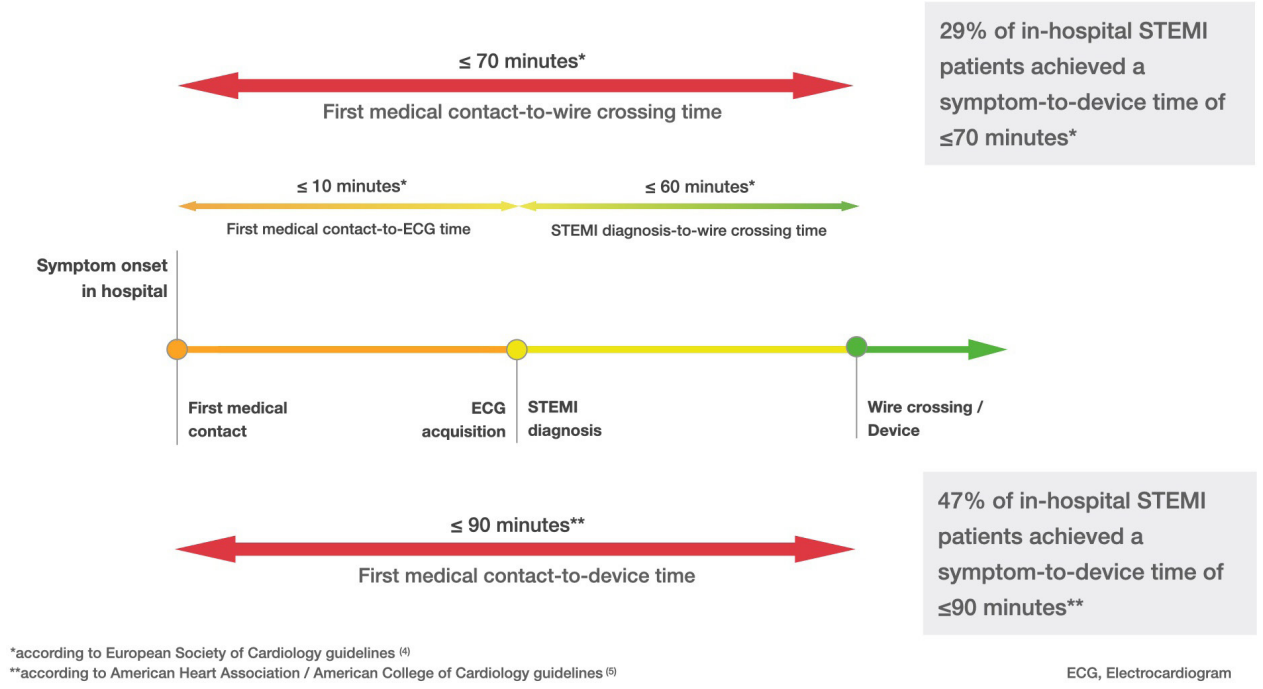

Figure 2 Proportion of patients with in-hospital ST-elevation myocardial infarction (STEMI) achieving guideline-recommended ischaemic times. Patients with in-hospital STEMI achieved a symptom-to-device (STD) of $\leq 70 \mathrm{~min}$ in $29 \%$ and an STD time of $\leq 90 \mathrm{~min}$ in $47 \%$.

due to delays in ECG acquisition and interpretation. ${ }^{617} \mathrm{In}$ order to reduce ischaemic times, education programmes of non-cardiac wards and medical staff should focus on reducing delays to ECG. ${ }^{22}$ Such education programmes should also highlight that in-hospital STEMIs may present with atypical symptoms, such as unexplained haemodynamic instability in the instance of postsurgical or intubated patients. ${ }^{23}$
We found that patients with in-hospital STEMI had a higher rate of bleeding, likely exacerbated by older age, higher use of oral anticoagulants, together with lower rates of radial access and perhaps due to surgical admissions. We know that postprocedural bleeding is a strong predictor of mortality in STEMI. ${ }^{24}$ The higher rates of bleeding and concomitant anticoagulant use in the in-hospital STEMI cohort may have led to the lower

\begin{tabular}{|c|c|c|c|c|c|c|c|}
\hline & $\begin{array}{l}\text { Overall } \\
\text { STEMI }\end{array}$ & $\begin{array}{l}\text { In-hospital } \\
\text { STEMI }\end{array}$ & $\begin{array}{l}\text { Out-of-hospital } \\
\text { STEMI }\end{array}$ & $\begin{array}{l}\text { Unadjusted } p \\
\text { value }\end{array}$ & $\begin{array}{l}\text { Adjusted } \\
\text { OR }\end{array}$ & $\begin{array}{l}\text { Adjusted } \\
95 \% \mathrm{Cl}\end{array}$ & $\begin{array}{l}\text { Adjusted } p \\
\text { value }\end{array}$ \\
\hline $\begin{array}{l}\text { 30-day } \\
\text { outcomes }\end{array}$ & $n=7493$ & $n=494$ & $\mathrm{n}=6999$ & & & & \\
\hline MACE, n (\%) & 785 (10.4\%) & 101 (20.4\%) & 684 (9.8\%) & $<0.001$ & 1.77 & 1.33 to 2.36 & $<0.001$ \\
\hline $\begin{array}{l}\text { All-cause } \\
\text { mortality, n (\%) }\end{array}$ & $542(7.2 \%)$ & $60(12.1 \%)$ & $482(6.9 \%)$ & $<0.001$ & 1.17 & 0.80 to 1.72 & 0.41 \\
\hline MACCE, n (\%) & 824 (11.0\%) & 108 (21.9\%) & 716 (10.2\%) & $<0.001$ & 1.88 & 1.42 to 2.49 & $<0.001$ \\
\hline $\begin{array}{l}\text { Major bleeding, } \\
\mathrm{n}(\%)\end{array}$ & $188(2.5 \%)$ & 24 (4.9\%) & $164(2.3 \%)$ & $<0.001$ & 1.81 & 1.13 to 2.89 & 0.01 \\
\hline $\begin{array}{l}\text { New heart } \\
\text { failure, } \mathrm{n}(\%)\end{array}$ & $112(1.5 \%)$ & 12 (2.4\%) & $100(1.4 \%)$ & 0.083 & 1.5 & 0.80 to 2.82 & 0.21 \\
\hline $\begin{array}{l}\text { Recurrent MI, } \\
\mathrm{n}(\%)\end{array}$ & $132(1.8 \%)$ & 28 (6.7\%) & $104(1.7 \%)$ & $<0.001$ & 3.23 & 2.01 to 5.20 & $<0.001$ \\
\hline $\begin{array}{l}\text { New renal } \\
\text { impairment, n } \\
(\%)\end{array}$ & $461(6.1 \%)$ & 53 (11.7\%) & $408(6.0 \%)$ & $<0.001$ & 1.53 & 1.09 to 2.16 & 0.01 \\
\hline $\begin{array}{l}\text { 12-month all- } \\
\text { cause mortality, } \\
n(\%)\end{array}$ & 718 (11.9\%) & 89 (22.5\%) & $629(11.2 \%)$ & $<0.001$ & 1.49 & 1.08 to 2.07 & 0.02 \\
\hline
\end{tabular}

Values are number (\%).

Adjustment was made for patient age, comorbidities, cardiogenic shock, intubation and out-of-hospital cardiac arrest, and time of symptom onset (day vs night).

MACCE, major cardiovascular and cerebrovascular events; MACE, major cardiovascular events; MI, myocardial infarction.; 
Kaplan-Meier survival estimates

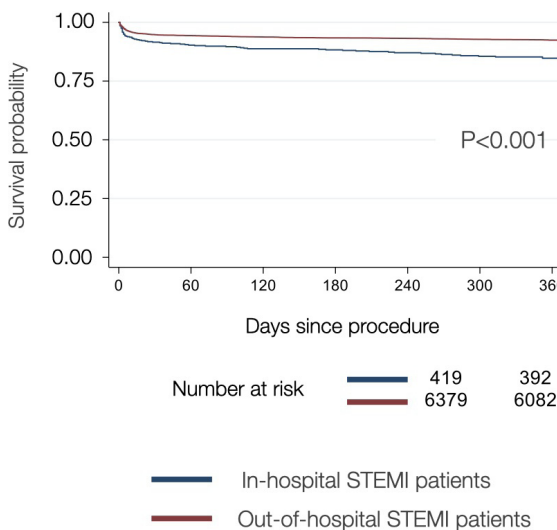

Adjusted* Kaplan-Meier survival estimates

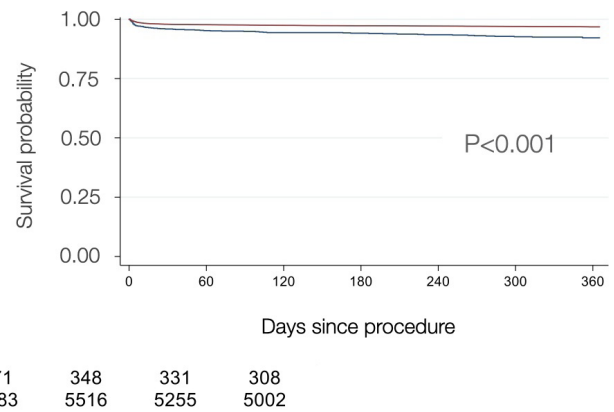

*adjusted for age, diabetes mellitus, history of coronary artery disease, cerebrovascular and peripheral artery disease, cardiogenic shock or

Figure 3 Unadjusted and adjusted Kaplan-Meier survival analysis. Twelve-months mortality was significantly higher for patients with in-hospital versus out-of-hospital ST-elevation myocardial infarction (STEMI) in the unadjusted and adjusted Kaplan-Meier survival analysis $(\mathrm{p}<0.001)$.

observed use of potent P2Y12 inhibitors. A total of 17\% of in-hospital STEMIs were due to stent thrombosis with 95\% unrelated to a recent PCI. It is possible that a major driving factor for in-hospital STEMIs was antiplatelet therapy cessation due to trauma or planned surgery. This again is likely to impact on optimal medical therapy and overall mortality. ${ }^{25}$ Of more concern is that significantly less patients with in-hospital STEMI received guidelinedirected medications at discharge, likely negatively impacting on MACE. ${ }^{26}{ }^{27}$ Potential explanations include patients with in-hospital STEMI being older, potentially frailer, with more comorbidities. However, these significant discrepancies have not been described before and warrant further study.

\section{Limitations}

Our study provides observational data and therefore only associations can be made between ischaemic time and outcomes. Further, our study is limited by the registry not capturing the condition for which patients with in-hospital STEMI were hospitalised, which could have influenced outcomes. However, this is similar to the few previous studies on in-hospital STEMI, where exact admission diagnoses were not known. ${ }^{6} 8$ In addition, we are limited in ascertaining where the delays in ischaemic times stem from, with time of ECG acquisition (and therefore ECG-to-device time) not routinely collected. We cannot exclude selection and survivor bias since only

\section{Central illustration: Patients with In-hospital STEMI Timeline of factors leading to worse outcomes}

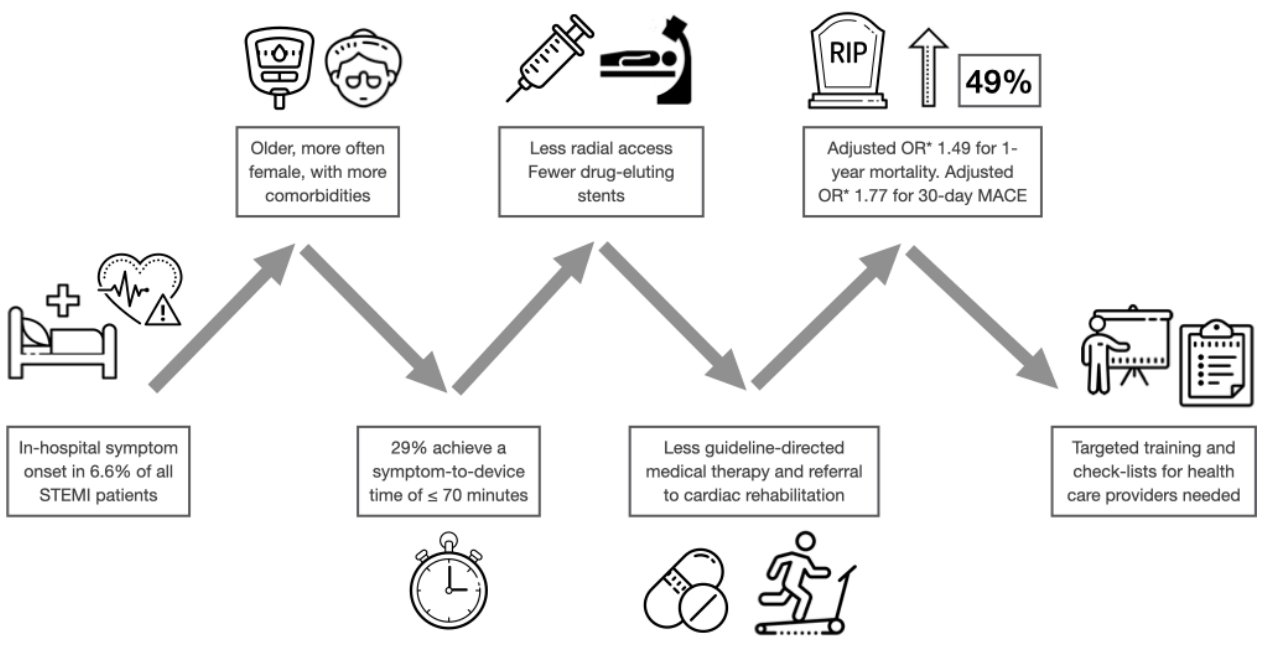

-Adjusted for age, comorbidities, left ventricular function, cardiogenic shock and/or cardiac arrest requiring intubation MACE: Major adverse cardiovascular event; OR: Odds ratio; STEMI: ST elevation myocardial infarction

Figure 4 Illustration of a series of factors influencing outcomes in patients with in-hospital ST-elevation myocardial infarction (STEMI). 
patients with STEMI treated with PCI were included. However, it is well known that only a minority of patients with in-hospital STEMI undergo PCI, which would only strengthen our findings, since in-hospital STEMIs not taken to the catheterisation laboratory are likely to have an even poorer outcome. Further, by studying a more homogenous population of patients with in-hospital STEMI, all deemed candidates for primary PCI, we have still identified significant treatment and outcome disparities. The data were not assessed for multiple recruitment; however, only $5 \%$ of stent thrombosis-related in-hospital STEMI occurred during the same admission as an initial PCI. Lastly, a number of patients had to be excluded from the data analysis, such as those who initially presented to non-PCI capable hospitals since VCOR only collects STD time and DTD time at hospital that are. However, these patients would have had to be excluded regardless since they would have introduced a large bias, representing a totally different patient cohort than those presenting directly to a PCI capable hospital.

\section{CONCLUSIONS}

Patients with in-hospital STEMI experience reperfusion delays and face significantly worse outcomes than patients with out-of-hospital STEMI, even after adjustment for confounders. We identified key targets to address, namely strategies to improve ischaemic time and optimal medical therapy prescription. Raising awareness of the issues facing patients who suffer a STEMI while already admitted to hospital is urgently needed if we are to improve outcomes in this under-investigated and under-treated patient population (figure 4).

\section{Author affiliations}

${ }^{1}$ Faculty of Medicine Nursing and Health Sciences, Monash University, Clayton, Victoria, Australia

${ }^{2}$ Epworth HealthCare, Richmond, Victoria, Australia

${ }^{3}$ Department of General Medicine, Alfred Health, Melbourne, Victoria, Australia

${ }^{4}$ Centre of Cardiovascular Research and Education in Therapeutics, School of Public Health and Preventive Medicine, Monash University, Clayton, Victoria, Australia

${ }^{5}$ Department of Cardiology, The Royal Melbourne Hospital, Melbourne, Victoria, Australia

${ }^{6}$ Cardiovascular Medicine, Alfred Hospital, Melbourne, Victoria, Australia

${ }^{7}$ School of Clinical Sciences at Monash Health, Monash University, Clayton, VIctoria, Australia

${ }^{8}$ Westmead Applied Research Centre, University of Sydney, Sydney, New South Wales, Australia

${ }^{9}$ Cardiology Department, Westmead Hospital, Westmead, New South Wales, Australia

Correction notice This article has been corrected since it was first published. The funding statement has been updated.

Twitter Julia Stehli @JStehli

Contributors JS, DTD and SZ are responsible for the overall content as guarantors. JS and SZ planned the project, created the hypothesis, drafted the manuscript and interpreted the results. MD, ALB, JL, SO, RD and SJD gave intellectual input and proofread the manuscript.

Funding Funding for this research was provided by the Epworth Medical Foundation.

Competing interests JS is supported by a Monash University scholarship. RD receives teaching and travel grants from Abbott, Medtronic and Boehringer. He serves on the advisory board of Astra Zeneca. SJD is proctor for Medtronic and his work is supported by a grant (1111170) from the National Health and Medical Research Council of Australia. SZ has obtained research funding from Abbott Vascular, Biotronik Australia and Medtronic Australia and speaking honoraria from AstraZeneca. All other authors have no conflicts of interest.

Patient consent for publication Not applicable.

Ethics approval This study complies with the Declaration of Helsinki and was approved by the locally appointed Institutional Human Research Ethics Committee (Monash Health, Monash Medical Centre, Victoria, Australia) with an opt-out consent.

Provenance and peer review Not commissioned; externally peer reviewed.

Data availability statement Data are available upon reasonable request. The deidentified data analysed for the purpose of this study are available upon request to the Victorian Cardiac Outcomes Registry Committee (email: vcor@monash.edu).

Supplemental material This content has been supplied by the author(s). It has not been vetted by BMJ Publishing Group Limited (BMJ) and may not have been peer-reviewed. Any opinions or recommendations discussed are solely those of the author(s) and are not endorsed by BMJ. BMJ disclaims all liability and responsibility arising from any reliance placed on the content. Where the content includes any translated material, BMJ does not warrant the accuracy and reliability of the translations (including but not limited to local regulations, clinical guidelines, terminology, drug names and drug dosages), and is not responsible for any error and/or omissions arising from translation and adaptation or otherwise.

Open access This is an open access article distributed in accordance with the Creative Commons Attribution Non Commercial (CC BY-NC 4.0) license, which permits others to distribute, remix, adapt, build upon this work non-commercially, and license their derivative works on different terms, provided the original work is properly cited, appropriate credit is given, any changes made indicated, and the use is non-commercial. See: http://creativecommons.org/licenses/by-nc/4.0/.

\section{ORCID iDs}

Julia Stehli http://orcid.org/0000-0003-3089-1062

Stephen J Duffy http://orcid.org/0000-0002-4518-5948

Sarah Zaman http://orcid.org/0000-0001-6289-583X

\section{REFERENCES}

1 Nowbar AN, Gitto M, Howard JP, et al. Mortality from ischemic heart disease. Circ Cardiovasc Qual Outcomes 2019;12:e005375.

2 Kristensen SD, Laut KG, Fajadet J, et al. Reperfusion therapy for ST elevation acute myocardial infarction 2010/2011: current status in 37 ESC countries. Eur Heart J 2014;35:1957-70.

3 Scholz KH, Maier SKG, Maier LS, et al. Impact of treatment delay on mortality in ST-segment elevation myocardial infarction (STEMI) patients presenting with and without haemodynamic instability: results from the German prospective, multicentre FITT-STEMI trial. Eur Heart J 2018;39:1065-74.

4 Ibanez B, James S, Agewall S, et al. 2017 ESC guidelines for the management of acute myocardial infarction in patients presenting with ST-segment elevation: the task force for the management of acute myocardial infarction in patients presenting with ST-segment elevation of the European Society of cardiology (ESC). Eur Heart $J$ 2018;39:119-77.

5 O'Gara PT, Kushner FG, Ascheim DD, et al. 2013 ACCF/AHA guideline for the management of ST-elevation myocardial infarction: a report of the American College of cardiology Foundation/American heart association Task force on practice guidelines. Circulation 2013;127:e362-425.

6 Dai X, Bumgarner J, Spangler A, et al. Acute ST-elevation myocardial infarction in patients hospitalized for noncardiac conditions. J Am Heart Assoc 2013;2:e000004.

7 Jaski BE, Grigoriadis CE, Dai X, et al. Factors associated with Ineligibility for $\mathrm{PCl}$ differ between inpatient and outpatient STelevation myocardial infarction. J Interv Cardiol 2016;29:363-9.

8 Kaul P, Federspiel JJ, Dai X, et al. Association of inpatient vs outpatient onset of ST-elevation myocardial infarction with treatment and clinical outcomes. JAMA 2014;312:1999-2007.

9 Townsend N, Wilson L, Bhatnagar P, et al. Cardiovascular disease in Europe: epidemiological update 2016. Eur Heart J 2016;37:3232-45.

10 Solomon MD, McNulty EJ, Rana JS, et al. The Covid-19 pandemic and the incidence of acute myocardial infarction. N Engl J Med Overseas Ed 2020;383:691-3. 
11 Shi S, Qin M, Cai Y, et al. Characteristics and clinical significance of myocardial injury in patients with severe coronavirus disease 2019. Eur Heart J 2020;41:2070-9.

12 Stefanini GG, Montorfano M, Trabattoni D, et al. ST-Elevation myocardial infarction in patients with COVID-19: clinical and angiographic outcomes. Circulation 2020;141:2113-6.

13 Stub D, Lefkovits J, Brennan AL, et al. The establishment of the Victorian cardiac outcomes registry (VCOR): monitoring and optimising outcomes for cardiac patients in Victoria. Heart Lung Circ 2018;27:451-63.

14 Cox N, Brennan A, Dinh D, et al. Implementing sustainable data collection for a cardiac outcomes registry in an Australian public hospital. Heart Lung Circ 2018;27:464-8.

15 Thygesen K, Alpert JS, Jaffe AS, et al. Fourth universal definition of myocardial infarction (2018). J Am Coll Cardiol 2018;72:2231-64.

16 Arzamendi D, Ly HQ, Tanguay J-F, et al. Differential 1-year clinical outcomes for ST-segment elevation myocardial infarction related to stent thrombosis or saphenous vein graft thrombosis. Catheter Cardiovasc Interv 2013;82:193-200.

17 Garberich RF, Traverse JH, Claussen MT, et al. ST-Elevation myocardial infarction diagnosed after hospital admission. Circulation 2014;129:1225-32.

18 Zahn R, Schiele R, Seidl K, et al. Acute myocardial infarction occurring in versus out of the hospital: patient characteristics and clinical outcome. maximal individual therapy in acute myocardial infarction (MITRA) Study Group. J Am Coll Cardiol 2000;35:1820-6.

19 Dai X, Kaul P, Smith SC, et al. Predictors, treatment, and outcomes of STEMI occurring in hospitalized patients. Nat Rev Cardiol 2016;13:148-54.
20 Yeh RW, Sidney S, Chandra M, et al. Population trends in the incidence and outcomes of acute myocardial infarction. $N$ Engl $J$ Med 2010;362:2155-65.

21 Erne P, Bertel O, Urban P, et al. Inpatient versus outpatient onsets of acute myocardial infarction. Eur J Intern Med 2015;26:414-9.

22 Dai X, Meredith D, Sawey E, et al. A quality improvement program for recognition and treatment of inpatient ST-segment elevation myocardial infarctions. JAMA Cardiol 2016;1:1077-9.

23 Levine GN, Dai X, Henry TD, et al. In-Hospital ST-segment elevation myocardial infarction: improving diagnosis, triage, and treatment. JAMA Cardiol 2018;3:527-31.

24 Valgimigli M, Costa F, Lokhnygina Y, et al. Trade-off of myocardial infarction vs. bleeding types on mortality after acute coronary syndrome: lessons from the thrombin receptor antagonist for clinical event reduction in acute coronary syndrome (tracer) randomized trial. Eur Heart J 2017;38:804-10.

25 Sorrentino S, Sartori S, Baber U, et al. Bleeding risk, dual antiplatelet therapy cessation, and adverse events after percutaneous coronary intervention: the Paris registry. Circ Cardiovasc Interv 2020;13:e008226.

26 Cannon CP, Braunwald E, McCabe CH, et al. Intensive versus moderate lipid lowering with statins after acute coronary syndromes. N Engl J Med 2004;350:1495-504.

27 Anderson L, Oldridge N, Thompson DR, et al. Exercise-Based cardiac rehabilitation for coronary heart disease: cochrane systematic review and meta-analysis. J Am Coll Cardiol 2016:67:1-12. 